\title{
A HIGHLY SENSITIVE IMPEDIMETRIC APTASENSOR FOR WEARABLE DETECTION OF HORMONES
}

\author{
Sanaz Pilehvar ${ }^{1 *}$, Shuyu Lin ${ }^{1 *}$, Hannaneh Hojaiji ${ }^{1}$, Yichao Zhao ${ }^{1,2}$, and Sam Emaminejad ${ }^{1}$ \\ ${ }^{1}$ Department of Electrical and Computer Engineering, University of California, Los Angeles, CA, USA \\ ${ }^{2}$ Department of Materials Science and Engineering, University of California, Los Angeles, CA, USA \\ * Equal Contribution
}

\begin{abstract}
A highly sensitive impedimetric aptamer-based sensing method is developed for the wearable detection of hormones, that are present in biofluids at nanomolar concentrations. The methodology is applied to monitor the cortisol diurnal profile in iontophoretically-induced sweat.
\end{abstract}

\section{INTRODUCTION}

Wearable sweat analysis offers a promising pathway toward non-invasive and continuous monitoring of hormones for a diverse set of clinical and personalized health monitoring applications. While previously reported sweat sensors successfully demonstrated on-body measurement of a panel of target analytes, because of the lack of suitable in-situ signal enhancement strategies, their detection limit remained in $\mu \mathrm{M}$ to $\mathrm{mM}$ range. To overcome this barrier, here, we devise and combine two synergistic sensor development strategies: 1) direct electrochemical detection of probe-target analyte based on DNA-aptamer-target biomarker interactions using electrochemical impedance spectroscopy (EIS) technique and 2) incorporation of a redox-probe treated protective layer which enhances the sensitivity of the EIS measurements, while preventing the sensor surface degradation caused by the inherent etching effect of redox probes. As a proof of concept, we applied this approach for sensitive and on-body detection of cortisol, which is present in nanomolar levels in biofluids (including sweat) and has promising clinical utility (as a key metabolic regulator and a stress marker).

\section{RESULTS AND DISCUSSION}

In our approach, the underlying EIS based sensing mechanism relies on monitoring the interfacial resistance change of the working electrode, which is measured in the presence of the reversible redox probe $\left[\mathrm{Fe}(\mathrm{CN})_{6}\right]^{3-} /\left[\mathrm{Fe}(\mathrm{CN})_{6}\right]^{4-}$.

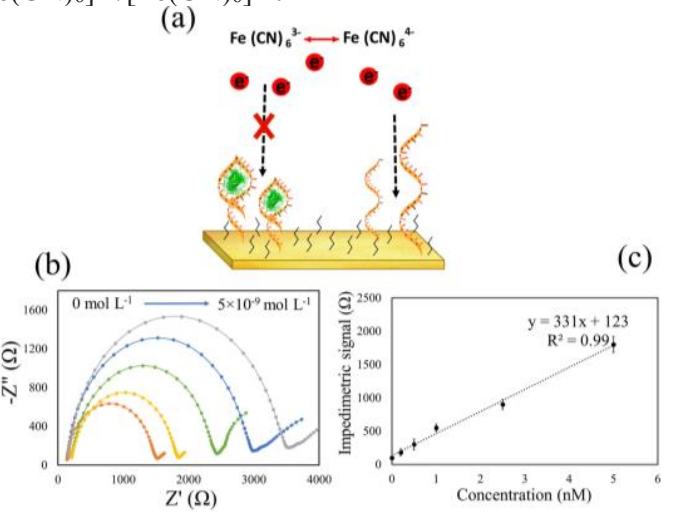

Figure 1: (a) Schematic diagram of the aptasensor operation, illustrating the partial blockage of electron transfer due to the formation of aptamer-target analyte complex, (b) EIS responses to different concentration of cortisol. (c) The sensor's corresponding calibration curve.

The sensing interface consists of DNA-aptamer-based cortisol recognition elements, which are covalently immobilized on the surface of a working electrode. The formation of aptamer-cortisol complex at the surface results in the partial blockage of electrontransfer (as shown in Fig. 1a). This leads to an increase in the interfacial resistance which is correlated to the concentration of cortisol (our corresponding measurement results are shown in Fig. $1 b, c)$. Additionally, to overcome the etching effect of the redox probes, we incorporate them into a protective layer mounted on the sensor surface. Here, the etching effect is induced by $\mathrm{CN}^{-}$released from the $\left[\mathrm{Fe}(\mathrm{CN})_{6}\right]^{3-} /\left[\mathrm{Fe}(\mathrm{CN})_{6}\right]^{4-}$ couple, which leads to gradual removal of the self-assembled monolayer of DNA probes [1].

(a)

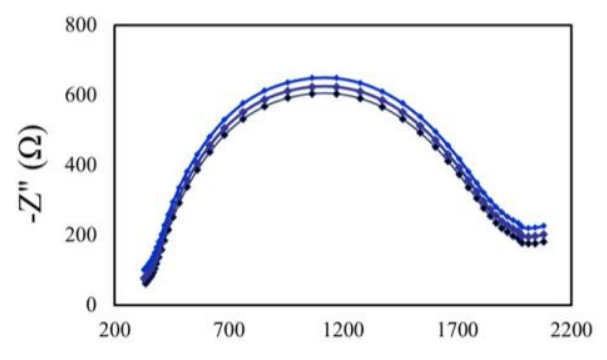

(b)

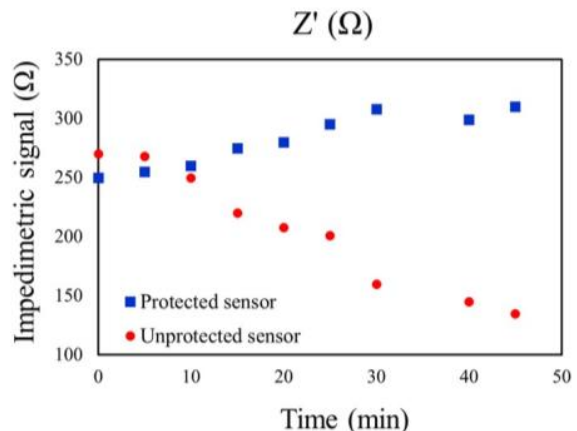

(c)

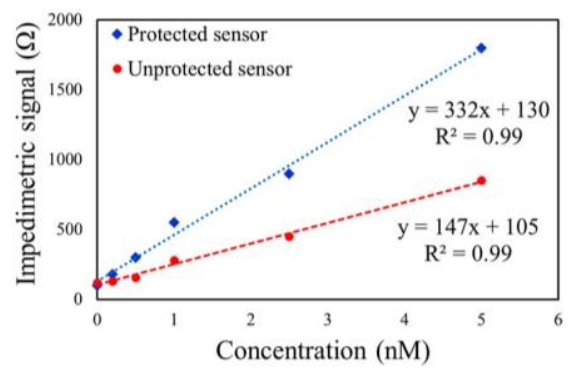

Figure 2: (a) Intermittent impedance measurements (Nyquist plots) with 10 min intervals, illustrating preserved stability of the sensing layer with the use of the protective layer. (b) Comparison of the protected vs. unprotected sensor response to a $5 \mathrm{nM}$ cortisol solution: the response of the unprotected sensing interface degrades over time. (c) Comparison of the protected vs. unprotected sensor calibration curve, illustrating the enhanced sensitivity of the protected sensor.

The incorporated protective layer preserves the sensing surface from direct exposure to $\mathrm{CN}^{-}$containing medium, while facilitating the originally intended signal transduction mechanism (Fig $2 \mathrm{a}-\mathrm{c}$ ). 
(a)

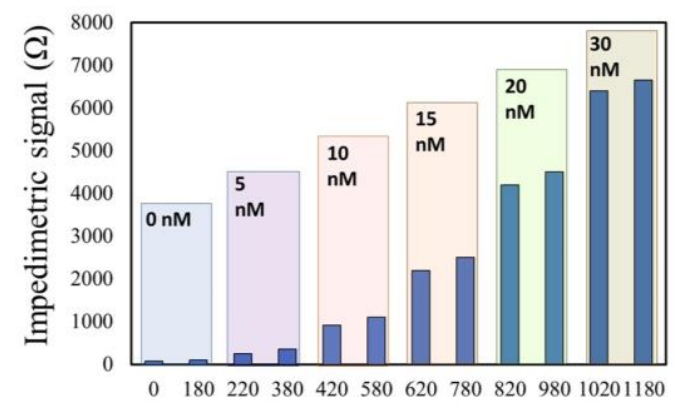

(b)

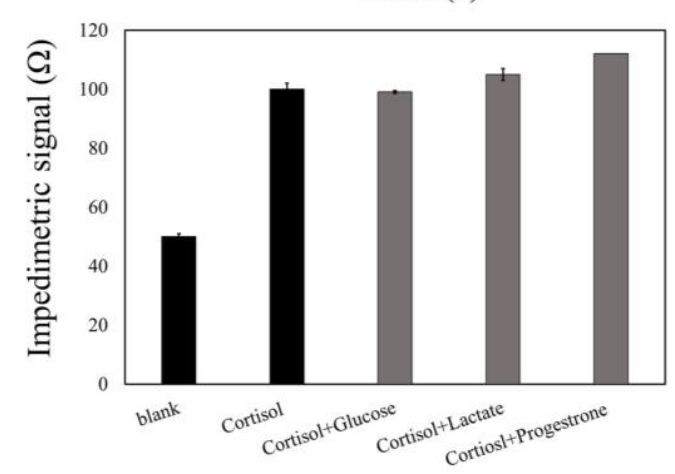

Figure 3: (a) Continuous sensor response characterization for progressively increasing cortisol concentration. (b) Aptasensor interference study: comparison of the sensor response to $5 \mathrm{nM}$ cortisol solution, to that with added interfering non-target analytes, including glucose $(100 \mu \mathrm{M})$, lactate $(100 \mu \mathrm{M})$ and progesterone (5 nM) (all spiked in Tris buffer).

To characterize our sensor, first, the continuous response of the sensor for different cortisol concentration regimes $(5,10,15$, and 20 $\mathrm{nM}$ ) is evaluated over time. As shown in Fig. 3a, our results indicate the preserved stability of the sensor's progressive response. Additionally, we performed interference studies to characterize the selectivity of the sensor to ensure that the non-target compounds in sweat (some with several orders of magnitude of higher concentration) do not interfere with the sensor response. To this end, we evaluated the sensor response against an illustrative panel of non-target analytes including glucose, lactate, and progesterone (within their physiologically relevant range of concentrations). The obtained data shows that non-specific bindings have minimal influence on the sensor response (as evident from small changes in the transduced signal, Fig $3 \mathrm{~b}$ ).

To inform the potential future clinical applications, the devised methodology was applied to analyze the cortisol content of iontophoretically-induced sweat samples, collected from a healthy subject during a day (Fig. 4a). We validated our sensor accuracy by comparing the concentration values, as estimated by our sensor response, with those obtained from High Performance Liquid Chromatography (HPLC, as a gold standard technique, Fig. 4b). The high degree of agreement for obtained measurements $\left(R^{2}=1\right)$ demonstrates our sensor's remarkable level of accuracy. The versatility of our methodologies allows for their use, with minimal effort and reconfiguration, to target various other hormones and metabolites in other non/minimally-invasive biofluids such as saliva, interstitial fluid, and urine. Therefore, we envision that the presented methodologies would open new directions for personalized health monitoring. (a)

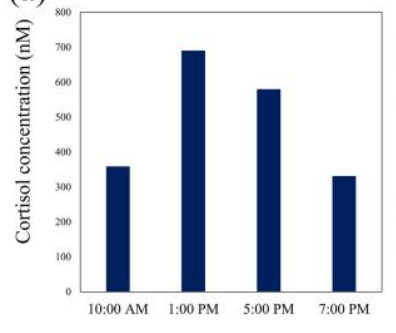

(b)

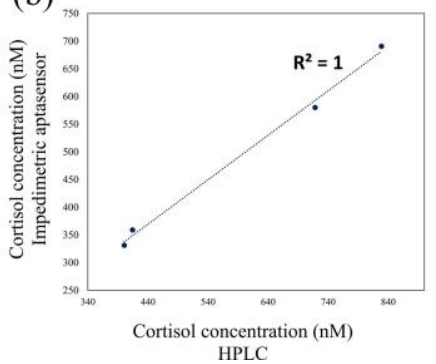

Figure 4: The diurnal sweat cortisol profile of a healthy subject, as measured by our developed aptasensor (a), The correlation of gold standard HPLC and our aptasensor, demonstrating our sensor's high level of accuracy (b).

The high degree of agreement for obtained measurements demonstrates our sensor's remarkable level of accuracy $\left(R^{2}=1\right)$. The versatility of our methodologies allows for their use, with minimal effort and reconfiguration, to target various other hormones and metabolites in other non/minimally-invasive biofluids such as saliva, interstitial fluid, and urine. Therefore, we envision that the presented methodologies would open new directions for personalized health monitoring.

\section{REFERENCES}

[1] S. Vogt, Q. Su, C. Gutiérrez-Sánchez, and G. Nöll, "Critical View on Electrochemical Impedance Spectroscopy Using the Ferri/Ferrocyanide Redox Couple at Gold Electrodes", Analytical chemistry, 88, 4384 (2016).

\section{CONTACT}

S. Emaminejad, emaminejad@ucla.edu 